\title{
The Nature of Force and the Dark Matter
}

\author{
Xingwu Xu \\ Research Institute of Hefei Guoxuan High-tech Power Energy Co., Ltd. 599 \\ Daihe Road, Hefei, Anhui 230012, P. R. China \\ E-mail addresses: xuxingwu@gotion.com.cn
}

Received Sept 2019

Received in revised: Sept 2019

Published: Sept 2019

\begin{abstract}
There are different approaches for investigating the force as well as the gravity. Newton emphasized the mathematical meaning of gravity. Einstein's gravity is geometry. The physical exploring for the gravity is still on the way. Le Sage investigated the nature of gravity, but his theory has many defects. This paper gives out a physical chemistry approach for the nature of force, which bases on gravitons' reaction by introducing the concepts of Gibbs free energy change and holographic screen. The change of the Gibbs free energy results in the imbalance on the two sides of the holographic screen, it is the force. The change of force as the distance depends on the distribution of gravitons. For solar system, the graviton gas distributes in center-radical pattern, so the inverse-square law is applicable. But in the galaxies, the distribution of the graviton gas is not in center-radical, therefore the acceleration is just inversely proportional to the distance (not square of distance), which is equivalent to the centripetal force. The flatness of rotation curve can be explained without dark matter.
\end{abstract}

Keywords: Nature of Force, Gravity, Gibbs free energy, Dark Matter

C2019 The Authors. Published by Fundamental Journals. This is an open access article under the CC BY-NC https://creativecommons.org/licenses/by-nc/4.0/

https://doi.org/10.14331/ijfps.2019.330130

\section{INTRODUCTION}

For a long time the nature of force, especially for the action at distance of the magical gravitational force, remain to be clearly answered even until now. There is a universal inclination that we need not to know about the nature of force because maybe there is not any influence on our application of the laws of force. Galileo said that he did not know the essence of the gravity (Jammer, 1999, pp. 53-54). For Newton, he derived the Inverse Square Law mathematically (Newton, Cohen, \& Whitman, 1999, p. 108), and he confessed that he considered these forces not from physical but only from a mathematical point of view (Newton et al., 1999, pp. 53-54). In the eyes of Einstein, gravity is geometry (Isaacson, 2007, p. 193). Besides the mathematical approach, research along the direction of physics is also under way (Feynman, Morinigo, \& Wagner, 
2003). The third approach is the kind of Le Sage's theory, which has a resurgent trend (Edwards, 2002). The purpose of this paper is to put forward the physical chemistry approach for the nature of force, which based on the Le Sage's theory. Once we make clear the nature of force, it is natural to solve the Dark Matter problem.

\section{THE PHYSICAL CHEMISTRY THEORY OF THE NATURE OF FORCE}

Here the graviton gas will be introduced to replace Le Sage's ultra-mundane corpuscles. In the shadow area between two bodies these graviton gas will make reaction. We still do not know the mechanism of this reaction. However, we can consider this reaction as a thermodynamic process. We just need to know the energy situation in original and last states. This is to say, we need to investigate the average energy change of graviton gas before and after reaction whatever in the shadow or in other sides of the bodies. The two important concepts will be introduced,

1) The concept of Gibbs free energy in graviton gas reaction.

2) The concept of holographic screen.

1- On the holographic screen the change of Gibbs free energy in two sides of the body will decide the motion's direction of the body, which is the discriminant of Gibbs free energy change (See Fig.1).

The attracting reaction will occur automatically

$$
\Delta \mathrm{G}=\Delta \mathrm{H}-\mathrm{T} \Delta \mathrm{S}<0
$$

The attracting reaction cannot occur automatically

$$
\Delta \mathrm{G}=\Delta \mathrm{H}-\mathrm{T} \Delta \mathrm{S}>0
$$

The system will be in the thermal equilibrium state

$$
\Delta \mathrm{G}=\Delta \mathrm{H}-\mathrm{T} \Delta \mathrm{S}=0
$$

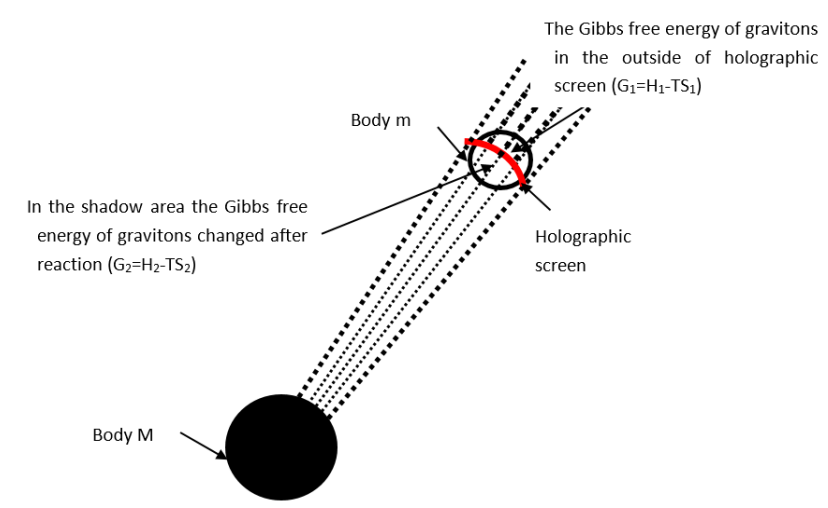

Fig.1 The Gibbs free energy changed after reaction in the shadow.

That is to say, if $\Delta G<0$ the two bodies will attract together automatically. In addition, if $\Delta G>0$ then the two bodies will repel each other. Let us investigate the reaction of gravitons in two different cases.

i) The generally accepted theory is that the spin of graviton is 2. Suppose during the reaction process the spin of gravitons is in same direction (Fig.2), let us analysis the
Gibbs free energy change in the two sides of the holographic screen,

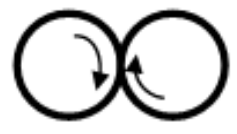

Fig.2 Diagram of the helical collision of two gravitons with same spin direction.

According to total differential form of Gibbs free energy,

$$
d G=d U+p d V+V d p-T d S-S d T
$$

Just to consider the fixed volume and isothermal process, we have

$$
d G=d U+V d p-T d S
$$

As shown in Fig. 2 the change of Gibbs free energy in the two sides of the holographic screen is

$$
\Delta G=G_{2}-G_{1}=\left(U_{2}-U_{1}\right)+V\left(p_{2}-p_{1}\right)-T\left(S_{2}-S_{1}\right)
$$

In this case, after reaction the average energy (angular momentum) of gravitons will decrease, which means $U_{2}<$ $U_{1}(\Delta U<0)$. Meanwhile, because the angular momentum of gravitons decreases, the pressure of graviton gas decreases, which means, $\left(p_{2}-p_{1}\right)=\Delta p<0$.

In the shadow area, because there is a reaction or collision between gravitons emitted from two bodies, the entropy becomes larger $(\Delta S>0)$. This situation is similar to the mixing process of two sets of gases. Therefore,

$$
\Delta G=\Delta U+V \Delta p-T \Delta S<0
$$

Therefore, the two bodies will attract each other automatically.

ii) For the condition that the spin of gravitons or photons is in different direction (Fig.3), after the helical collision the average energy (angular momentum) of gravitons or photons keeps unchanged, which means $\Delta U=0$. In the shadow area, the number of gravitons or photons is larger than that of other side of the holographic screen, and because the average angular momentum of gravitons or photons is the same, the pressure of graviton gas or photon gas in the shadow side is larger than that in other side of the holographic screen. That is $\left(p_{2}-p_{1}\right)=\Delta p>0$. Here we suppose that the pressure of graviton gas or photon gas dominates the reaction (from the result that the same pole of magnet repels, this assumption is reasonable), which means $\Delta p V>T \Delta S$. Therefore, the change of Gibbs free energy is,

$$
\Delta G=\Delta H-T \Delta S>0
$$

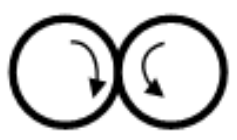

Fig.3 Diagram of the collision of two gravitons or photons with different spin direction.

The two bodies will repel each other. This is the situation of two same poles of an electrical magnet ( $\mathrm{N}-\mathrm{N}$ poles). 
Moreover, if we change the direction of current in one of the electromagnets, the spin direction of photons emitted from one of the electromagnet will change too, the two objects will become attractive. However, we cannot do the same thing for the gravitational force at present unless the anti-matter found.

2- The concept of the holography and the holographic screen. In 1947 Dennis Gabor, a Hungarian, invented the holography. The basic principle is that the light wave has recorded by using the interference principle, which not only the amplitude in the image surface (or film) has recorded, but also the amplitude as well as the phase in 3D space recorded. In this way, the 3D image of the light wave can be reproduced.

The word hologram was coined by Gabor, the Greek word holo means whole (Potcoava, 2009, p. 1). Therefore, the holography is the technology, which the information of 3D space can be reflected by $2 \mathrm{D}$ recording plane. The foundation of holography is the application of Gauss theorem into the record of light wave. In fact, the Gauss theorem is just to convert the information of $3 \mathrm{D}$ space into the $2 \mathrm{D}$ surface

$$
\begin{aligned}
\iiint_{V}\left(\frac{\partial P}{\partial x}+\frac{\partial Q}{\partial y}\right. & \left.+\frac{\partial R}{\partial z}\right) d V \\
& =\oint_{S}(P \cos \alpha+Q \cos \beta+R \cos \gamma) d S
\end{aligned}
$$

According to the wave equation

$$
\Psi(x, y, z, t)=u(x, y, z) e^{-i \omega t}
$$

Where $u(x, y, z)$ is the complex amplitude of the wave and $\exp (-i w t)$ is the wave absolute phase time variation. The Gauss theorem can be applied to the vector fields $\mathrm{U}$ and $\mathrm{V}$ (Potcoava, 2009, pp. 24-25).

$$
\int_{V}\left(U \nabla^{2} V+V \nabla^{2} U\right) d V=\oint_{S}(U \nabla V+V \nabla U) d S
$$

Where $U$ and $V$ are any two complex-valued functions of position, $S$ is the closed surface surrounding a volume $V, \nabla=$ $\partial / \partial n$ is the partial derivative in the outward normal direction at each point on $S$. Equation(11) is the basic equation of the holography. It shows that the information in $3 D$ space can be reflected by the information in $2 D$ holographic screen. For the reaction of graviton gas, we can imagine a holographic screen cutting through the mass-center point, the reacting information of the graviton gas in the shadow can be reflected in the holographic screen: attracting or repelling, the strength of force etc.

Therefore, the states of gravitons (e.g. the areal density of gravitons, the Gibbs free energy change of graviton gas etc.) in the holographic screen are closely related to the force exerting to the body. This is the microscopic explanation for the nature of force. There is no action at distance here. In the holographic screen, the reaction of gravitons or photons instantaneously implemented. Moreover, if there is an energy difference in the two sides of the holographic screen there will be a motion, whatever attractive or repulsive. That is the root cause of motion, and that is the force. In Le Sage's theory, one of the issues is that there is no relation between the gravitational force and the masses of bodies. This problem can be solved as follows.
First, let us discuss the number of gravitons and the Gibbs free energy change of gravitons after reaction. We know that the change in Gibbs free energy is equal to the virtual work made by the gravitational force,

$$
G_{\text {shadow }}=F \cdot r=G_{\text {gravity }} \frac{M m}{r}
$$

Here $\Delta G_{\text {shadow }}$ is the change of Gibbs free energy in shadow area $(J / \mathrm{mol}), \mathrm{M}$ and $\mathrm{m}$ are the masses of larger and smaller bodies respectively, $\mathrm{r}$ is the distance between two bodies, $G_{\text {gravity }}$ is the gravitational constant. As we known, the value of Gibbs free energy is unknown. It is enough to know the change of Gibbs free energy. Here a relative simple method will be taken to treat this problem. Assuming that the average change of Gibbs free energy of graviton gas $(\Delta g)$ is only expressed by the change of average frequency of the gravitons, thus,

$$
\Delta g=h \Delta v
$$

Here $h$ is Planck constant, $\Delta v$ is the change of average frequency. Now the concept of mole number of gravitons of standard or special condition, will be introduced, which is similar to the mole number of gas. The mole number is denoted as $N_{m}$,

$$
G_{\text {shadow }}=N_{m} \Delta g=N_{m} h \Delta v=G_{\text {gravity }} \frac{M m}{r}
$$

For two bodies with the masses of $1 \mathrm{Kg}$ respectively, $1 \mathrm{~m}$ distance between them, the change of frequency is $1 s$, the mole number of gravitons is

$$
N_{m}=\frac{G_{\text {gravityMm }}}{r h \Delta g}=\frac{G_{\text {gravity }}}{h}=10^{23}
$$

This result shows that the mole number of gravitons is in the same order of magnitude of Avogadro's number in the special condition (the ratio of gravitational constant and Plank constant, $G / h)$. Meanwhile, it means that the gravitational force is proportional to the mass of the body. As a comparison, Le Sage's theory of gravity as well as the similar theory has to face two difficulties.

1) The aberration as well as the force couple issue. Because there is a propagation speed of the ultramundane corpuscles, there will be a transit delay and aberration phenomenon.

2) The over-heat issue. Because of the high speed of the corpuscles, there will be a lot of heat generated from the collision of the ultramundane corpuscles.

For issue (1), according to Van Flandern (1998, pp. 1-11), it is necessary for gravity that its propagation speed must be $\geq 2 \times$ $10^{10} c$, where $c$ is the light speed. It is unacceptable (Carlip, 2000; Marsh \& Nissim-Sabat, 1999, pp. 257-260). However, in the theory of this paper, the reaction of gravitons in the holographic screen accomplishes instantly, which means that there will be no aberration. For issue (2), as pointed out by Maxwell, $M u^{2}$ ( $M$ is mass, $u$ is velocity of the ultramundane corpuscles) must be enormous compared with the corresponding quantity for ordinary bodies, and it follows that the impact of the corpuscles would raise all bodies to an enormous temperature (Edwards, 2002, p. 69). However, it is not the case in the theory of this paper, because the graviton is massless (no $M$ term). This theory about the nature of force can perfectly describe the gravitational force (attraction) as well as the electromagnetic force (repulsion and attraction). Moreover, it is very useful in explanation of the dark matter. 


\section{UNDERSTANDING OF DARK MATTER FROM THE VIEWPOINT OF NEW THEORY}

The Dark Matter issue originated from the estimating matter density in our galactic neighbourhood in the first half of the last century (Luković, Cabella, \& Vittorio, 2014). In October 1937 Fritz Zwicky published the paper titled "On the Masses of Nebulae and Clusters of Nebulae" (Zwicky, 1937). In this paper, F. Zwicky made use of the virial theorem which based on the Newtonian Dynamics to estimate the masses of nebulae. F. Zwicky gave two important assumptions,

(1) Newton's inverse square law accurately describes the gravitational interaction between nebulae.

(2) The nebulae has a central, highly viscous heavy core. According to the Newton Second Law

$$
M_{\sigma} \frac{d^{2} \boldsymbol{r}_{\sigma}}{d t^{2}}=\boldsymbol{F}_{\sigma}
$$

Where $r_{\sigma}$ is the radius vector from a fixed point in the cluster to the nebulae $(\sigma)$ of mass $M_{\sigma}, F_{\sigma}$ is the total force acting on $M_{\sigma}$. From equation, (16) the Virial Theorem can be derived

$$
\frac{1}{2} \frac{d^{2} \theta}{d t^{2}}=V_{i r}+2 K_{T}
$$

Where $\theta=\sum_{\sigma} M_{\sigma} r_{\sigma}^{2}$ is the polar moment of inertia of the cluster, $V_{i r}=\sum_{\sigma} \mathbf{r}_{\sigma} \cdot \mathbf{F}_{\sigma}$ is the virial of the cluster, $K_{T}$ is the sum of the kinetic energies of translation of the nebulae. If the cluster is stationary, the polar moment of inertia will remain unchanged with time. So we get,

$$
V_{i r}=2 K_{T}
$$

The average mass of nebulae in Coma cluster calculated by virial theorem is

$$
\bar{M}>4.5 \times 10^{10} M_{\Theta}
$$

However, that mass estimated by luminosity is around $8.5 \times$ $10^{7} M_{\Theta}$, which is less than around 500 times. This result is unexpected. Therefore, there must be a lot of dark matter in the nebulae. In 1970, Vera C. Rubin and W. Kent Ford Jr. researched the relationship between the velocity and the distance from the center of the Andromeda nebula. The velocities of stars were determined by the $H_{\alpha}$ spectral line emitted by hydrogen gas clouds that had been ionized by nearby hot stars. They found that the rotational velocity curve is flat, which means that the rotational velocity changes less with the increase of distance to center (Fig.4) (Rubin \& Ford Jr, 1970).

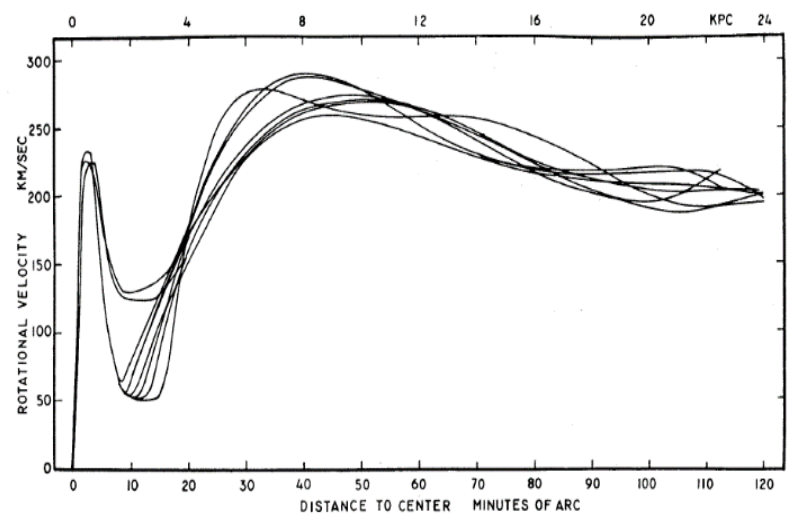

Fig.4 Fourteen rotation curves for M31(Rubin \& Ford Jr, 1970)
This phenomenon does not obey the law of Keplerian fall-off,

and

$$
V(R) \propto R^{-\frac{1}{2}}
$$

$$
V^{2}=G \frac{m}{R}
$$

Therefore, there must be a lot of dark matter to speed up the rotational velocities of these stars, or compensates the mass (m) with the increase of R. This is one of the main arguments of Dark Matter. However, according the new theory of this paper, it is important to analyse the variation of gravitons' areal density in the holographic screen as well as the Gibbs free energy change in the two sides of the holographic screen. In the center-radial gravitational field, the gravitons' areal density (i.e. the gravitational flux density in Gauss theorem) is inversely proportional to the square of distance. The gravitational field which obeys Gauss theorem is certainly in accordance with Newton Second Law. Now let's investigate the application of Gauss theorem in the galaxy and compare the difference between the applications in galaxy and solar system. Regarding the application of Gauss theorem into the galaxy, there are two different viewpoints at present. Gauss theorem is invalid in galaxy (Capozziello \& Stabile, 2009; Stabile \& Scelza, 2011). The Gauss theorem of gravitational field shows that the gravitational flux through any closed surface is proportional to the enclosed mass (Capozziello \& Stabile, 2009). But according to $f(R)$ theory, especially in the galaxy, the Green function which has the meaning of pointsource overlying does not satisfy the Gauss theorem. Because the matter outside the sphere of integration does not contribute to the gravitational flux. The no viability of Gauss theorem implies that the range of integration of dark matter (DM) could cover all range and also the matter outside is considered. A cutoff radius is needed.

(2) The Gauss theorem is better than Poison's equation in the application of the galaxy (Hofmeister \& Criss, 2017). Hofmeister et al. consider that, such dark matter has not be detected despite expenditures of billions of dollars over several decades, motivating alternative models. The computationally intensive force calculation is an alternative to using Poisson's equation. These three independent studies using force calculations have shown that non-baryonic matter is negligible for certain density distributions. Importantly, Feng and Gallo showed that the force at any given radius is derived from mass both inside and outside this radius. Because the density parameter cannot sum, and the mass can sum, therefore the Gauss theorem is better than Poison's equation in application to the galaxy. Meanwhile, the theorem of Gauss directly specifies the interior mass, whereas the Poison's equation does not consider the contribution of outside matter to the gravitational potential. The gravitational potential for threebody problem is,

$$
U_{g, 3 b o d y}=\frac{G m_{1} m_{2}}{r_{1}-r_{2}}-\frac{G m_{2} m_{3}}{r_{2}-r_{3}}-\frac{G m_{3} m_{1}}{r_{3}-r_{1}}
$$

Where $G$ is the gravitational constant, $m_{1}, m_{2}$, and $m_{3}$ are individual masses, $r_{1}, r_{2}$, and $r_{3}$ are distances of particle separations. Orbits in this system are unlike Keplerian. Let's back to the issue of application of Gauss theorem. The Gauss theorem of gravitational field is 


$$
\Phi_{\text {gravity }}=\oint_{S} g d S=-4 \pi G \sum_{i=1}^{n} m_{i}
$$

Equation (23) shows that the gravitational flux $\Phi_{\text {gravity }}$ through the closed surface $S$ is proportional to the algebraic sum of enclosed masses, and is not affected by the masses outside the surface $S$. Where the intensity of the gravitational field $g$ is,

$$
g=\frac{G M}{r^{2}} e
$$

Here $e$ is the unit direction vector. For the situation of uniform distribution of gravitational flux, the intensity of the gravitational field is the gravitational flux per unit area (the areal density of gravitons)

$$
g=\frac{\Phi_{\text {gravity }}}{S}
$$

In the gravitational field with center-radial pattern (e.g. the solar system, the earth etc.), the gravitational flux areal density (the density of gravitons) is inversely proportional to the square of distance r, as shown in Fig.5

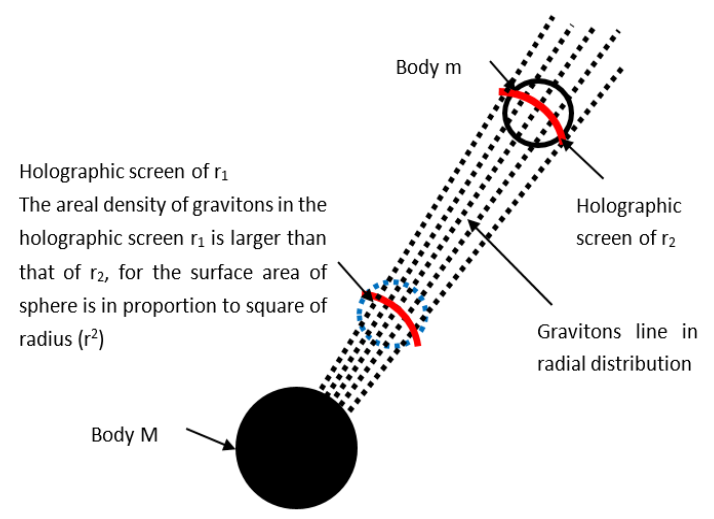

Fig.5 The gravitational force is proportional to the area density of gravitons in holographic screen, thus inversely proportional to the square of distance $r$.

The mathematic expression is,

$$
g \propto \frac{1}{r^{2}}
$$

This is a parabolic curve. However, in the galaxy the distribution of gravitons does not have to be in center-radical. In fact, these gravitons in a galaxy will interfere and interweave each other which is very similar to the magnetic field of U-magnet (Fig.6 ).

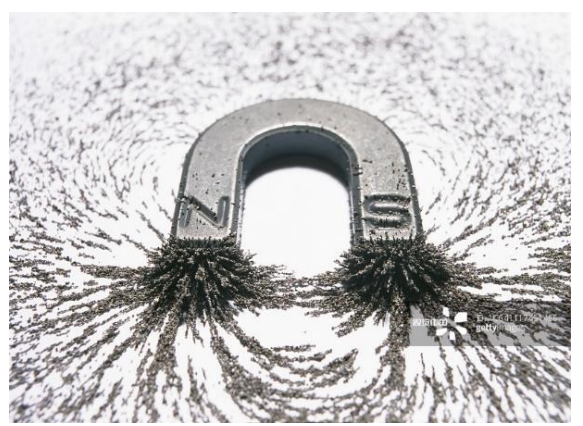

Fig.6 The distribution of magnetic field of the U-magnet
For a small segment of electric current, the photons of magnetic field distribute in center-radial pattern, the magnetic field is inversely proportional to the square of distance (BiotSavart law). Whereas for many electric segments, the photons will interfere and interweave each other, as a result, the distribution of photons must not be in center-radial pattern. Therefore, the magnetic force does not obey the inverse square law here. To investigate the distribution of gravitons in the galaxy, we can do a thought experiment: every planet emits its gravitons in center-radial pattern, and many planets' gravitons will interfere and interweave together. The pattern is shown in Fig. 7

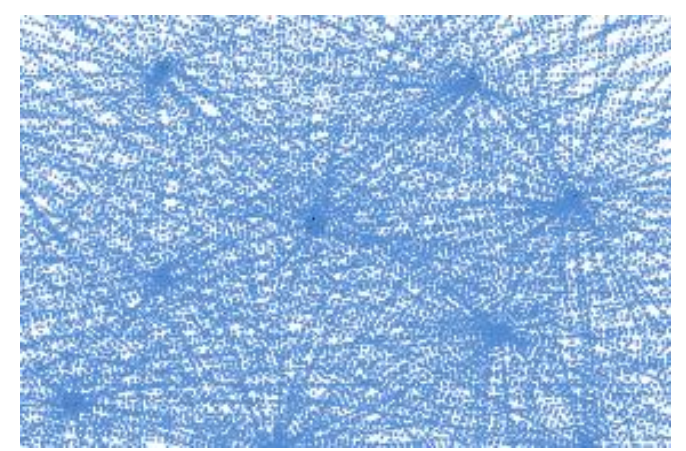

Fig.7 The gravitons' distribution after the interfered and interweaved

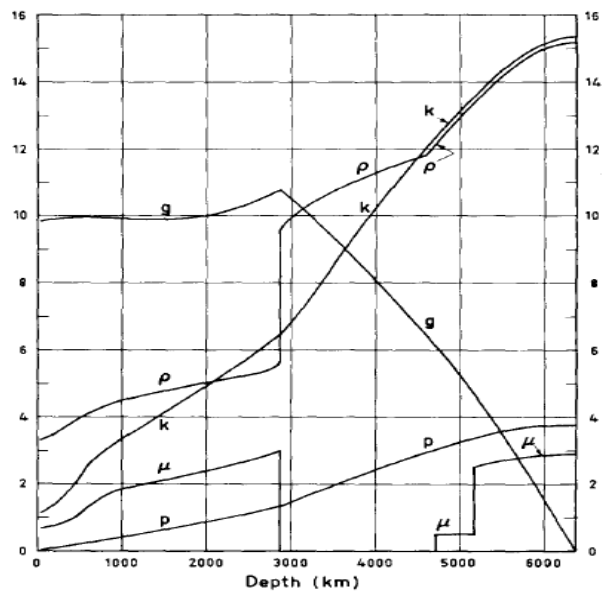

Fig.8 Variation of $\rho$ and $g$ in Earth model $B_{2}$ in the galaxy (thought experiment) (Bullen \& Haddon, 1967).

Therefore, the enclosed mass within Gauss's surface (holographic screen) is a variable. The change pattern of gravitational field in the galaxy must be different with the solar system in which the gravitational field is in center-radical distribution. We can compare the galaxy with the distribution of gravitational field in the interior of the earth.

According to Bullen's model (Bullen \& Haddon, 1967) and data of the earth's density, the distribution of gravitational field as well as the mass density shown in Fig.8.

We note that the variation of gravitational acceleration $g$ as the depth in the interior of the earth is very complex. It is not a parabolic curve. According to Gauss theorem, the variation of gravitational field intensity as the distance in the galaxy is complex.

Nevertheless, it is sure that the variation of the areal density of gravitons or gravitational flux as the distance in Gauss surface or the holographic screen is different. One possibility is that 
the gravitational field intensity of the galaxy is just inversely proportional to the distance $r$ (not the square of distance).

$$
g \propto \frac{1}{r}
$$

It is just like the situation in Fig. 8 for the curve corresponding to the depth of $3000 \sim 6000 \mathrm{Km}$.

Therefore, the acceleration $g \propto 1 / r$ is equivalent to the centrifugal acceleration $v^{2} / r$, the rotation curve is flat. From the viewpoint of shadow of gravitons as well as the Gibbs free energy change, let's see what the difference between the galaxy and the solar system is.

In fact, in the non-center-radial gravitational field (e.g. the galaxy), there is no shadow for the distribution of gravitons surrounding the body, or everywhere is the shadow. The gravitons emitted from all directions interweave together to form a large web. It is the sticky web that the planets in galaxy are combined together to a whole.

Assuming that the Gibbs free energy change of the sticky gravitons which take part in gravitational reaction is a constant, as shown in Fig. 9.

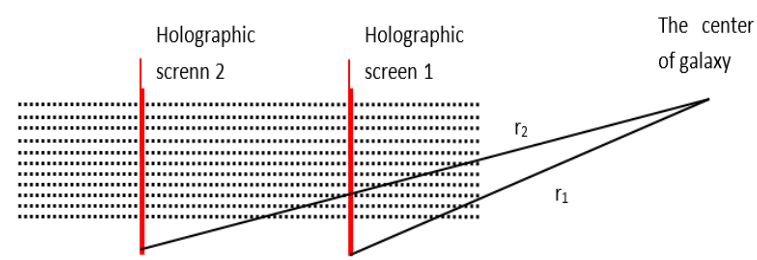

Fig.9 The areal densities of gravitons are almost equal both in holographic screen 1 and holographic screen 2

Thus, we have,

$$
G_{\text {shadow }}=C
$$

According to equation (12), we have

$$
F=\frac{G_{\text {shadow }}}{r}=\frac{C}{r}
$$

It is also proved that the rotation curve is flat. Equation (29) shows that Newton second law is not applicable in the galaxies. The gravitational force is not inversely proportional to the square of distance, but just inversely proportional to the distance. It is the basic requirement for the flat rotation curves of the galaxies that the Gibbs free energy change on the holographic screen keeps unchanged.

Only in this way the galaxy can maintain steady, whatever the part or the whole. It is worthwhile to emphasize that the phenomenon of almost no obvious change in rotation speed of planets does not violate the conservation law of momentum. It is similar to the atoms in a steel plate, that the rotation speed of atoms is same does not violate the conservation law of momentum.

Hofmeister and Criss (2017, pp. 1-21) treat the galaxies as the spinning solid objects in which internal coherency decreases with radius. Meanwhile, the analogy made between the spiral galaxies and the hurricanes.

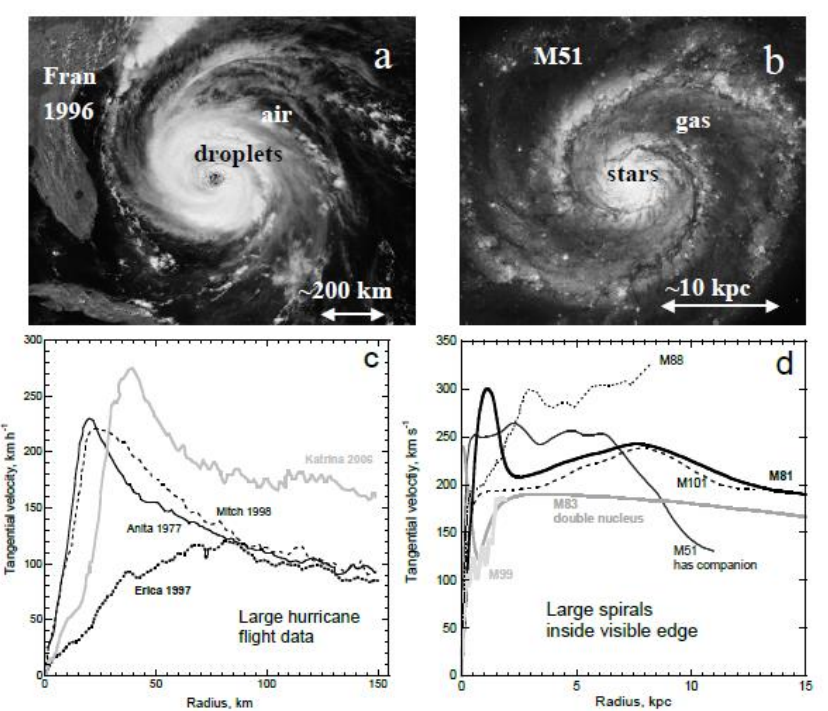

Fig.10 Comparison of hurricanes to the spiral galaxies.

(a) Image of hurricane (b) Image of the Whirlpool galaxy (c) Rotation curves for four large hurricanes (d) Rotation curves for complex spirals. (Hofmeister \& Criss, 2017)

From Fig. 10 we can see that there is an amazing similarity between the spiral galaxies and the hurricanes. It shows that the rotation curves for large hurricanes is still flat, which is similar to the solid body. In the disk-shaped galaxy, the formation of spiral patterns has been a mystery.

In 1964 C. C. Lin and Frank H. Shu put forward a theory of density waves. There is an important assumption in this theory as; there exists a spiral gravitational field in the galaxy. It is the spiral gravitational field that can forms the density waves, which keeps the steady of the spiral structure in the galaxy (Lin \& Shu, 1964).

According to the theory of this paper, that there is few change of Gibbs free energy in the spiral structure is every sides is the foundation of the spiral gravitational field. This argument afford an auxiliary support proof for the theory of this paper. As we known, the MOND theory does not give out the physical explanation for revised Newton's second law.

The theory of this paper can explain why the gravitational force is just proportional to the inverse of distance $(r)$ in the galaxy, as well as can give out the microscopic description. In fact, the essence of the revising is that the distribution of gravitons changes in solar system and the galaxy. Moreover, different galaxies have different pattern of the gravitons' distribution, this is why they have different rotation curves.

\section{CONCLUSIONS}

This paper gives out a new approach to investigate the nature of force, which is different from the mathematical approach (Newton) including the geometric approach (Einstein), the physical approach (Feynman et. al), as well as the pushing gravity theory (Le Sage et. al). This is a physical chemistry approach. Here the concept of Gibbs free energy change is very useful. The gravitons can be treated as a kind of "gas". In the shadow similar to Le Sage's theory, these gravitons will make reaction. By introducing the concept of holographic screen, according to the total differential equation of Gibbs free energy, $d G=d U+V d p-T d S$, the Gibbs free energy change $\Delta G$ of the graviton gas' reaction can be determined. The difference of the Gibbs free energy between the 
holographic screen is the force. Meanwhile, the attractive or repulsive force can also be determined. That the force changes with the distance is related with the distribution of gravitons. In the center-radial pattern of the gravitons distribution, the force obeys the inverse-square law.

However, in the no-center-radial pattern of the gravitons distribution (e.g. the galaxies), the areal density of gravitons in the holographic screen is not proportional to inverse-square of distance, and it keeps unchanged with the change of distance. Therefore, the acceleration $a$ is just proportional to the inverse of distance $r$ (not inverse-square of $r$ ), which is equivalent to the centripetal force. From above the Tully-Fisher, relation can also be derived. This relation shows that Newton second law is not applicable in the galaxies.

\section{REFERENCES}

Bullen, K., \& Haddon, R. (1967). Earth models based on compressibility theory. Physics of the Earth and Planetary Interiors, 1(1), 1-13.

Capozziello, S., \& Stabile, A. (2009). The Newtonian limit of metric gravity theories with quadratic Lagrangians. Classical and Quantum Gravity, 26(8), 085019.

Carlip, S. (2000). Aberration and the speed of gravity. Physics Letters A, 267(2-3), 81-87.

Edwards, M. R. (2002). Pushing gravity: new perspectives on Le Sage's theory of gravitation: Montreal: Apeiron.

Feynman, R. P., Morinigo, F. B., \& Wagner, W. G. (2003). BOOK REVIEW: Feynman Lectures on Gravitation. European Journal of Physics, 24, 330.

Hofmeister, A., \& Criss, R. (2017). Implications of Geometry and the Theorem of Gauss on Newtonian Gravitational Systems and a Caveat Regarding Poisson's Equation. Galaxies, 5(4), 89.

Isaacson, W. (2007). Einstein: His life and universe: Simon \& Schuster Audio, New York, USA.

Jammer, M. (1999). Concepts of force: A study in the foundations of dynamics: Courier Corporation.
This is why the gravitational force is just inversely proportional to the distance and the rotation curves of planets in the galaxy keep flat. The so-called dark matter is just the apparent effects of the distribution of gravitons. The steady of the spiral pattern in the disk-shaped galaxy can afford an auxiliary proof of the new theory of this paper. Further, the mechanism of the reaction of graviton gas needs to be investigated. The distribution of gravitons in the galaxies needs to be detected too. One of the prediction of the new theory is that the distribution of gravitons in the galaxies is not in center-radial pattern. If one day the distribution of gravitons can be detected out, the new theory will be proved to be correct. Moreover, this theory is very simple. As Chandrasekhar pointed out (1984, pp.147). The simple is the seal of the true.

Lin, C., \& Shu, F. H. (1964). On the spiral structure of disk galaxies Selected Papers of CC Lin with Commentary: Vol. 1: Fluid Mechanics Vol. 2: Astrophysics (pp. 561-570): World Scientific.

Luković, V., Cabella, P., \& Vittorio, N. (2014). Dark matter in cosmology. International Journal of Modern Physics A, 29(19), 1443001.

Marsh, G. E., \& Nissim-Sabat, C. (1999). Comment on "The speed of gravity". Physics Letters A, 262(2-3), 257-260.

Newton, I., Cohen, I. B., \& Whitman, A. (1999). The Principia: Mathematical Principles of Natural Philosophy: University of California Press.

Potcoava, M. C. (2009). Digital holography applications in ophthalmology, biometry, and optical trapping characterization.

Rubin, V. C., \& Ford Jr, W. K. (1970). Rotation of the Andromeda nebula from a spectroscopic survey of emission regions. The Astrophysical Journal, 159, 379.

Stabile, A., \& Scelza, G. (2011). Rotation curves of galaxies by fourth order gravity. Physical Review D, 84(12), 124023.

Van Flandern, T. (1998). The speed of gravity - what the experiments say. Physics Letters A, 250(1-3), 1-11.

Zwicky, F. (1937). On the Masses of Nebulae and of Clusters of Nebulae. The Astrophysical Journal, 86, 217 